\title{
Research on Optimization Planning Method of Distribution Network based on Spatial Load Forecast
}

\author{
Shen Zeyuan ${ }^{1 *}$, Wang Ai ${ }^{1}$, Wu Jianbin ${ }^{1}$, Zhao Haibo ${ }^{1}$, Tian Liang ${ }^{1}$, Li Qi ${ }^{1}$, Qiu Jue ${ }^{1}$, Song Xiaojun ${ }^{1}$ and Zhi Shenglong ${ }^{1}$ \\ ${ }^{1}$ Economic and Technical Research Institute of SEPC of SGCC, Taiyuan, China
}

\begin{abstract}
Power grid planning is an important part in the development of power grid. With the rapid development of urban construction and electricity demand in recent years, electric power construction is faced with more kinds of pressure, such as land resources/environmental protection. In this paper, the difficulty of power grid planning is increasing rapidly. How to build a distribution network that the load and the urban construction can develop concordantly has become a key issue to be solved in the future. The traditional methods of distribution network planning focus on the grasp of the overall, whereas lack adequate consideration of the spatial distribution of load and planning of urban development. Thus, this paper proposes a method based on spatial load forecasting of urban distribution network planning g, and fully considers the load distribution of the space characteristics and urban construction. This method can make up for the defects of traditional methods effectively, thus has certain value and reference significance.
\end{abstract}

\section{Introduction}

Power grid planning is an important part of grid development and it is also a complicated system [1]. In recent years, with the rapid development of economy and society, urbanization in China has improved quickly, thus electricity demand of urban areas has been increased continuously and rapidly, which brings heavier pressure to the development of power grid. On the other hand, with the development of cities, the land resources have reduced gradually. Grid expansion is faced with lots of constraints such as land resources, environmental protection, and construction funds and so on. It is much more difficult to implement the planning of grid [2, 3]. How to build the distribution network coordinated with the development of the load and the overall layout of urban development has become a key problem in the planning of distribution networks.

The traditional planning method of distribution network thinks a way of 'from top to down', which determine the total demand and grid structure of distribution network through the supply capacity and distribution of the main network. This method is simple and easy to operate, but lacks the adequate consideration of the spatial distribution of the load and the planning of urban development. Although it focuses on the overall planning, it lacks the attention on local characteristics. This paper proposes a planning method based on spatial load forecasting. According to the spatial distribution of the land load and fully considering the planning of urban roads development, the overall layout of medium voltage grid can be designed. Based on the power supply area of high voltage substation, the capacity and location of substation can be decided. Unlike the traditional method which focuses on the total forecast planning, this method completely started from the load, from details to overall layout, from basic to top, which not only plans the total number accurately, but also fully considers the load, feeders and the distribution of the high voltage substation.

\section{The overall planning idea}

The object of this paper is urban distribution network. The research method is based on spatial load forecasting. As shown in Figure 1, the main research ideas include three parts which are load forecasting, medium voltage planning and high voltage planning.

The load forecasting part mainly adopts spatial load forecasting method and other forecasting methods are used to check and correct the predict results. Based on the results of load forecasting, planning is divided into medium voltage planning and high voltage planning. The medium voltage planning mainly include grid planning which lay cables along the road in the entire planning areas according to the city planning and the distribution of land load. On the basis of medium voltage grid, high voltage planning mainly determines the proper position for high voltage substation to insert into the medium voltage grid. With some flexibility, the power supply capacity requirements of the high voltage are determined through supply areas.

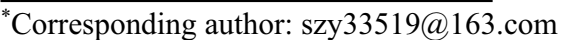




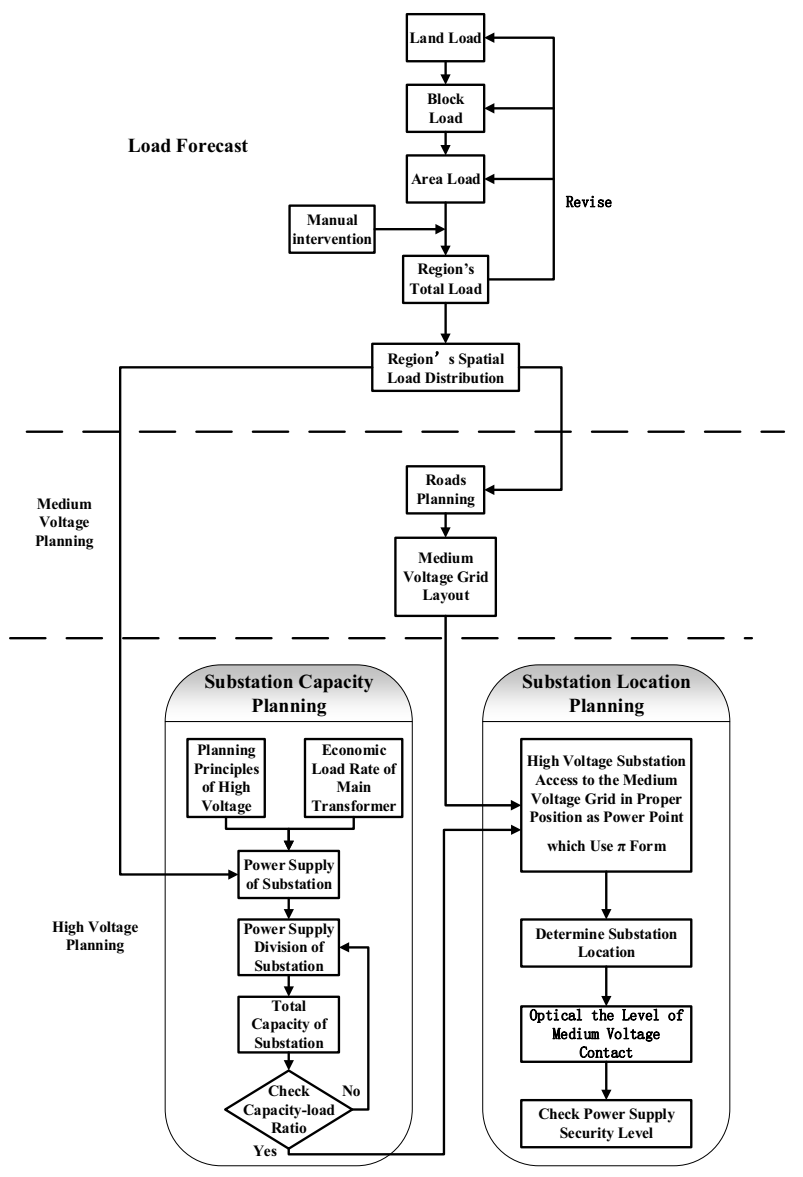

The rest of the paper is organized as follows: Section 2 load forecasting. Section 3 the planning of medium voltage. Section 4 the planning of high voltage. Section 5 one example of planning. The conclusions are concluded in section 6 .

\section{Load forecasting}

\subsection{Comparative analysis of various load forecasting methods}

Load forecasting is the basic work of distribution network planning. The accurate results of load forecasting are the foundation of scientific planning.

The most common methods of load forecasting are consumption value method, elastic coefficient method, time series, load dichotomy, spatial load forecasting method and so on. According to different forecast period, load forecasting is divided into short-term forecast (within 5 years), medium-term forecast (5-10 years) and long-term forecasts (10-20 years). According to different forecast contents, load forecasting can be divided into the total forecast and the spatial distribution forecast.

Figure 1. Figure of the overall planning idea.

Table 1. Comparison of Forecasting Methods

\begin{tabular}{|c|c|c|c|}
\hline $\begin{array}{c}\text { Forecasting } \\
\text { Method }\end{array}$ & $\begin{array}{c}\text { Forecast by content } \\
\text { category }\end{array}$ & $\begin{array}{c}\text { Forecast } \\
\text { period }\end{array}$ & Features \\
\hline $\begin{array}{c}\text { consumption } \\
\text { value method }\end{array}$ & total forecast & mid-term & $\begin{array}{c}\text { It is simple and easy to calculate, but the prediction results rely } \\
\text { much on the accuracy of GDP prediction, which is suitable for } \\
\text { system load forecasting and it can also be used to check the } \\
\text { results. }\end{array}$ \\
\hline $\begin{array}{c}\text { elastic coefficient } \\
\text { method }\end{array}$ & total forecast & long-term & $\begin{array}{c}\text { It is simple and easy to calculate, but the prediction results rely } \\
\text { much on the accuracy of GDP prediction, which requires a lot of } \\
\text { meticulous research and it can also be used to check the load } \\
\text { results. }\end{array}$ \\
\hline time series & total forecast & short-term & recent \\
load dichotomy & total forecast & $\begin{array}{c}\text { It is suitable for the area where large industrial users account for a } \\
\text { large proportion, and industrial users have a greater impact on the } \\
\text { results of load forecasting. }\end{array}$ \\
\hline $\begin{array}{c}\text { spatial load } \\
\text { forecasting }\end{array}$ & spatial prediction & long-term & $\begin{array}{c}\text { It can take full consideration of the spatial distribution of the load } \\
\text { characteristics, which is suitable for the areas which have the } \\
\text { planning of urban land and the planning of saturation annual load } \\
\text { forecast. }\end{array}$ \\
\hline
\end{tabular}

\subsection{Load forecasting method adopted in this paper}

This paper is based on the spatial load forecasting. On the basis of detailed city planning, it can respectively forecast land load, block load and area load in a way of "from down to top". With considering certain coincidence rate, ultimately the region's total load can be calculated. To compensate for spatial load forecasting defects, the total amount of prediction methods is used in the meantime, such as load dichotomy, elasticity coefficient, trend extrapolation and other methods. By integrating various methods and inserting manual intervention during the spatial load forecasting, the accuracy of predicted results will be improved.

As shown in Figure 1, ultimately the load of the total area and the detail load distribution of every area, street and block are available. 


\section{The planning of medium voltage network}

The main task of the medium voltage planning is the arrangement of $10 \mathrm{kV}$ feeder.

According to the distribution of the block load, $10 \mathrm{kV}$ feeders are arranged along the city roads under the principle that load of each $10 \mathrm{kV}$ line should carry no more than 6MW [4] and the block load need to be evenly distributed along the feeder. Throughout the planning area, the line will start with the load, extend with roads and finally cover the overall medium voltage grid, which ensures that every block load can have access to $10 \mathrm{kV}$ grid conveniently and uniformly.

Medium-voltage grid planning needs to take into consider several factors, including urban road planning, whether it can meet the power requirements of each block, the convenience of load access and load distribution and so on. And it is also taken into consideration to arrange the feeder as short as possible which can also meet the power requirements of block. Medium voltage grid planning is relevant with the actual situation of city and grid, which need professionals to carefully filter on and compare with a variety of plans.

\section{The planning of high voltage network}

The main object of high voltage network planning is an issue of determining the capacity and location in $110 \mathrm{kV}$ and $35 \mathrm{kV}$ substation.

\subsection{Substation capacity and power supply area planning}

In the traditional planning methods, the determination of substation capacity is based on the load forecasting results and capacity-load ratio, which belongs to the total forecast method. In this article, the substation capacity planning takes the thinking way of "from bottom to top" and gives full consideration to the spatial distribution characteristic of the load.

First of all, according to the load distribution and the main transformer power supply capacity, several power supply zones are divided reasonably in the whole planning area partition.

According to main transformer capacity specification and the best economic operation load rate, the load of the transformer ${ }^{P_{N}}$ is determined, as shown in the following equation:

$$
P_{N}=n \times S_{N} \times \varphi \times \beta
$$

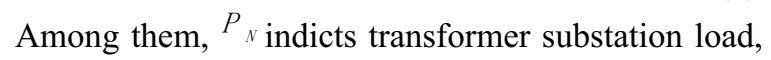
$S_{N}$ indicts transformer substation rated capacity, $n$ indicts the number of transformer units, $\varphi$ indicts the power factor, $\beta$ indicts the transformer load rate. It should be noted that the load rate of the main transformer should not only be close to the level of economic operation of the main transformer, but also take into account the requirement of $\mathrm{N}-1$ level of the medium voltage power grid, which is related to the load transfer capacity of the $10 \mathrm{kV}$ network.

According to the results of spatial load distribution, the planning area is divided into a number of load blocks of ${ }^{P_{N}}$, and plan under the principle that a load block corresponds to a substation.

1) For the New-constructed District, combined with blocks and streets distribution, load planning area is divided into a plurality of $P_{N}$ size block. Under the principle of a load block configuration corresponding to a substation planning, the power supply area of substation is determined.

2) For built-up old area, we must fully understand the present situation of power supply division and load distribution. According to the main transformer power supply capacity, the power supply division is optimized.

Therefore, according to the load distribution, the whole planning area is divided into a number of load blocks, each of which is the power supply area of a substation. According to the principle of a load block corresponding to one substation, the capacity of main transformer units can be obtained. Combined with regional total load forecasting, we conduct capacity-load ratio calibration, which must meet "the requirements of the distribution network planning technique guidelines". If they don't meet the guide, adjusts must be made according to actual situation of planning.

\subsection{Substation location planning}

Combined with the feeders' layout of MV network in the third part, we must look for the most appropriate location for substation to insert to the MV network. The determination of substation position should take full account of the grid structure, the contact-rate, and the situation of main grid.

After the determination of high voltage substation location, different feeders in one substation should be interconnected properly, meanwhile, feeders of adjacent substations should also be interconnected, so as to ensure the medium voltage network interconnection level and load transfer ability.

It is worth noting that after determining the location of high voltage station and the structure of medium voltage network, the $\mathrm{N}-1$ level of the whole medium voltage power network should be checked. In the second part, the load of the $10 \mathrm{kV}$ line is not exceed $6 \mathrm{MW}$, which ensures that the medium voltage circuit has $50 \%$ load transfer capacity, and it can protect the power security level of the power grid.

\section{Planning examples}

The planning area is Fendong business district, Taiyuan of Shanxi province. This region is located in South of Taiyuan, Xiaodian District, and the East Bank of Fen River. It locates in the key sections of core zone at southern part of Taiyuan city. The total area of Fendong business district is 3079.64 hectares, with population of 320 thousand people. 


\subsection{Spatial load forecast}

Using the spatial load density method to predict the future saturation load, on the basis of block load forecasting, the area load forecasting is carried out, and the total amount of the load forecasting is $430.11 \mathrm{MW}$. Combined with the load dichotomy, we take into account the situation of large users and intervene in the planning results, in the end, the regional total load forecasting in future planning will reach $800.11 \mathrm{MW}$. The regional control plan map is as follows.

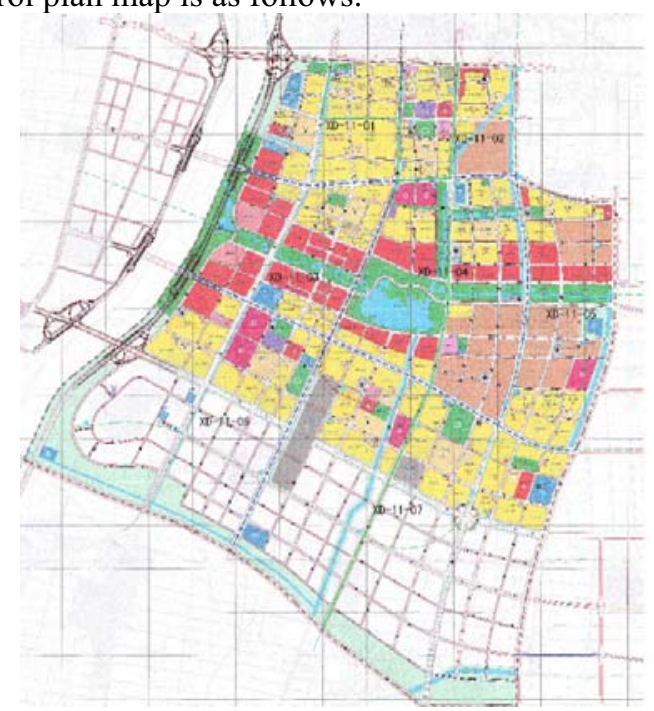

Figure 2. The regional control plan map

\subsection{Medium voltage network planning}

Based on block load forecasting results, according to a $10 \mathrm{kV}$ line with the principle of no more than $6 \mathrm{MW}$ load, we pave $10 \mathrm{kV}$ feeder along the city road, as shown in the figure below:

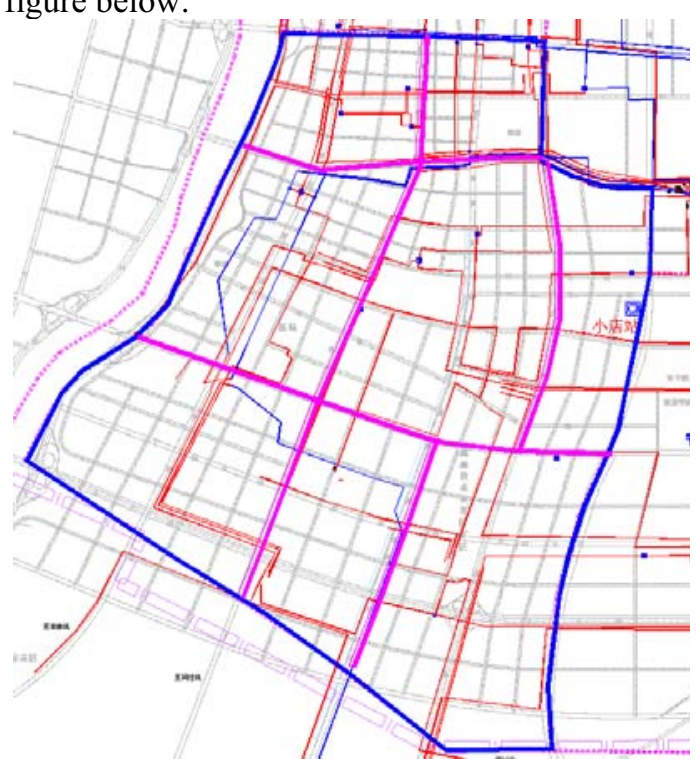

Figure 3. Distribution of medium voltage feeder

\subsection{High voltage network planning}

\subsubsection{Power supply area division}

The future of power grid in Taiyuan will no longer consider constructing new $35 \mathrm{kV}$ substation, so the planning substation only considers the $110 \mathrm{kV}$ voltage level.

According to the "guidelines for the distribution network planning technology", in the same planning area, the same voltage level of the main transformer capacity of a single specification should not be more than three, the same substation main transformer should be unified specifications. In this case, all the new substation adopts $3 * 63$ MVA transformer capacity configuration mode and the power factor $\varphi$ is considered as 0.8. According to the thirteenth five-year distribution network plan of Taiyuan, in the case of any $110 \mathrm{kV}$ substation full stop, all or the main load must be transferred by $10 \mathrm{kV}$ lines of other substations without the basic loss of power load. In this case, the main transformer load rate doesn't consider the N-1 level, instead it takes the economical operating load rate of $65 \%$ [4], by substituting it into formula (1), it can be calculated that the load of the substation should not exceed $98 \mathrm{MW}$.

According to the spatial load forecasting results, the load distribution is sorted out, and the whole planning area is divided into 8 zones, as is shown in the following figure.

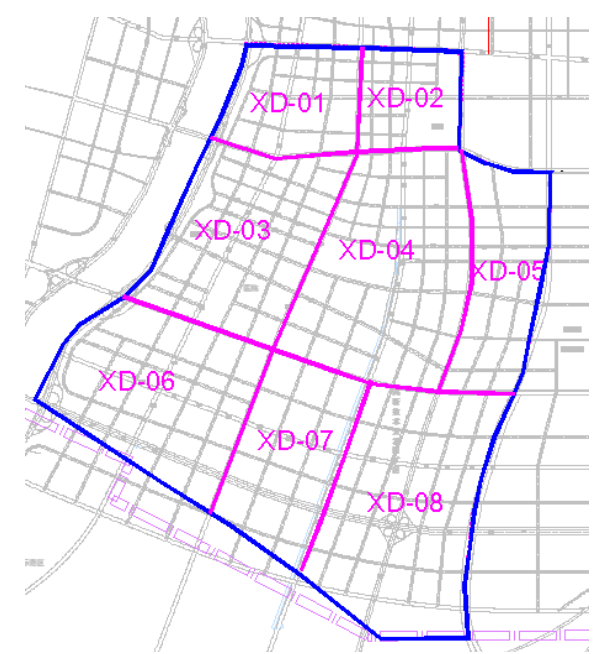

Figure 4. Power partition map

\subsubsection{Substation location planning}

Combining the layout of the distribution feeder in figure 3 , in each power supply area, we find the most suitable location to insert substation into medium voltage network, and establish interconnection between the feeders of the substation as well as feeders from adjacent substations, so as to ensure the interconnection level and load transfer capability of the medium voltage network. 


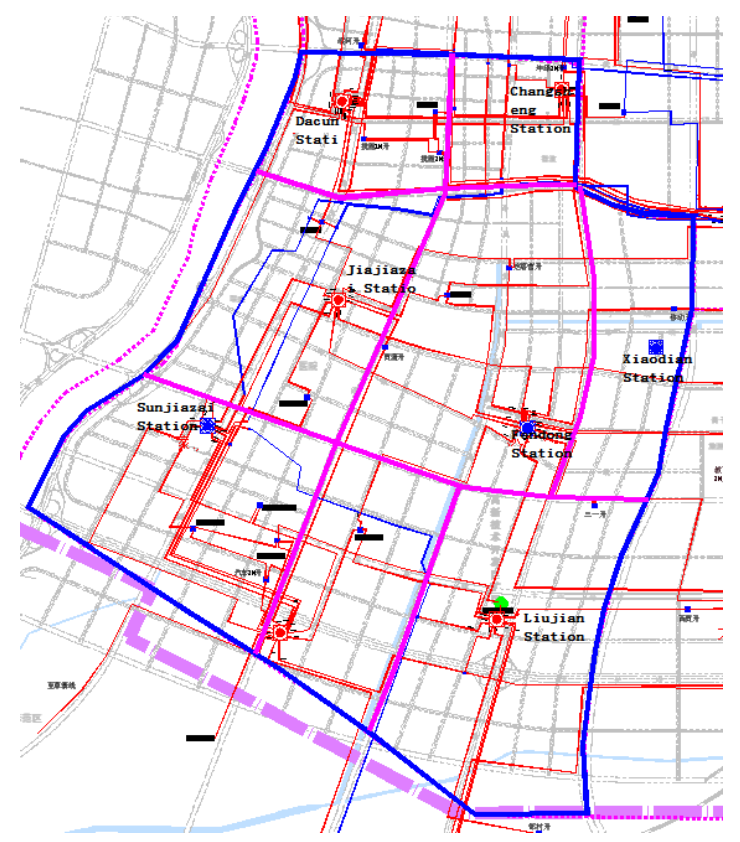

Figure 5. Visionary planning

\section{Conclusion}

- Different from traditional methods, in this paper, both the medium voltage feeder arrangement and high voltage substation capacity and location are totally based on load distribution. Medium voltage feeders are arranged starting with load and are along the road alignment, while substation are considered as a power generation to insert into medium voltage feeder in appropriate position, and the substation capacity is determined by the supply area which is divided by the load distribution. Traditional planning methods focused more on the total determination, but this method can fully consider the load characteristics and distribution, which adopts arranging wiring (medium voltage feeder), and then determining supply point (high voltage substation), to a certain extent, in this method, substation site selection is more flexible and convenient. In the modern urban distribution network, under the background of increasingly difficult in engineering project, this method has a certain meaning and reference value.

- Urban distribution network planning is a complex systematic project with the challenges of large amount of data, the existence of many uncertain factors and low information level. Under this circumstance, how to achieve a more efficient distribution network planning still needs a lot of work. In the future, we must do more in the development of power grid planning platform, so as to facilitate the electrical calculation, the verification of power supply security level, economic and technical comparison and so on, thus provide further scientific and reasonable assurance to the distribution network planning.

\section{References}

1. Yixin Yu, Gang Duan. Cognitive characteristics and methods of urban power grid planning [J].
Automation of electric power systems, 2001, 25 (2): 27-31.

2. Yi Song, Shaojie Ouyang, Xuyang Wang, Yali Wang, Ming Zeng. Considering the demand side resources of the new power grid planning model [J]. Power construction, 2013, 11:12-16.

3. Jun Xiao, Ting Zhang, Yue Zhang, Chengshan Wang. The idea and method of distribution network planning based on the maximum power supply capacity $[\mathrm{J}]$. Proceedings of the electrical engineering of China, 2013, 33 (10):106-113.

4. Q/GDW 1738-2012. "Technical guidelines for distribution network planning and design".

5. Xiao B,Liu T. A spatial load forecasting method based on load regularity analysis. Turkish Journal of Electrical Engineering\&Computer Sciences . 2017.

6. DAG Vieira,MMA Cabrall,T V Mnezes,et al. Measuring the Spatial Errorin Load Forecasting for Electrical Distribution Planning as a Problem ofTransporting the Surplus to The In-deficit Locations. 2012 11th International Conference on Machine Learning and Applications. 2012.

7. Zhu Fengjuan, Wang Zhuding, Lu Jian, et al. Disequilibrium development areas based classification and subarea method for spatial load forecasting $[\mathrm{J}]$. Automation of Electric Power Systems,2012, 36(12):41-47.

8. Xiao Bai, Zhou Chao, Mu Gang.Review and prospect of the spatial load forecasting methods[J]. Proceedings of the CSEE, 2013, 33(25):78-92.

9. Wang Chengshan, Xiao Jun, Luo Fengzhang. Interval based multi-layer decomposed calibration method for spatial load forecasting $[\mathrm{J}]$. Automation of Electric Power Systems, 2004, 28(12):12-17.

10. Wu HC, Lu, CN.A data mining approach for spatial modeling in small area load forecast[J]. IEEE Trans on Power Systems, 2002, 17(2):516-521.

11. Kang Chongqing, Xia Qing, Zhang Boming. Review of power system load forecasting and its development[J]. Automation of Electric Power Systems, 2004, 28(17):1-11.

12. Liu Zifa, Pang Chengcheng, Wei Jianwei,etal. Index calculation of load density based on IAHP and TOPSIS method[J]. Automation of Electric Power Systems, 2013, 36(13):56-60.

13. Li Jinchao, Niu Dongxiao, Li Jinying,etal. Spatial load forecasting based on load decomposition and support vector machine [J]. Advanced Technology of Electrical Engineering and Energy, 2008, 27(1):4043.

14. Wang Tianhua, Wang Pingyang, Fan Mingtian. Application of genetic algorithm, fuzzy logic and transshipment model to spatial load forecasting for distrbution planning[J]. Power System Technology, 1999, 23(1):24-28. 